\title{
Digital Image Denoising Techniques Based on Multi-Resolution Wavelet Domain with Spatial Filters: A Review
}

\author{
Ahmed Abdulmaged Ismael*, Muhammet Baykara \\ Department of Software Engineering, Technology Faculty, Firat University, Elazig 23119, Turkey
}

Corresponding Author Email: ahm.sula@gmail.com

https://doi.org/10.18280/ts.380311

Received: 28 February 2021

Accepted: 25 April 2021

\section{Keywords:}

digital image denoising, hybrid denoising, multi-resolution wavelet domain, spatial domain filtering, thresholding techniques

\begin{abstract}
Recently, with the explosion in the number of digital images captured every day in all life aspects, there is a growing demand for more detailed and visually attractive images. However, the images taken by current sensors are inevitably degraded by noise in various fields, such as medical, astrophysics, weather forecasting, etc., which contributes to impaired visual image quality. Therefore, work is needed to reduce noise by preserving the textural, information, and structural features of the image. So far, there are different techniques for reducing noise that various researchers have done. Each technique has its advantages and disadvantages. In this paper, a review of some significant work in the field of image denoising based on that the denoising methods is presented. These methods can be classified as wavelet domain, spatial domain, or both methods can combine to obtain the advantage them. After a brief discussion, the classification of image denoising techniques is explained. A comparative analysis of various image denoising methods is also performed to help researchers in the image denoising area. Besides, standard measurement parameters have been used to compute the results and to evaluate the performance of the used denoising techniques. This review paper aims to provide functional knowledge of image denoising methods in a nutshell for applications using images to provide ease for selecting the ideal strategy according to the necessity.
\end{abstract}

\section{INTRODUCTION}

The digital world is demonstrating people's real lives where it merged with every single aspect of our daily lives. Part of this world is the digital images that play an essential role in day-to-day applications such as digital photography, entertainment media, satellite imagery, medical imaging analysis, and remote sensing, as well as in fields of research and technology, including Geographical Information System (GIS) and Astronomy, Meteorology, Industrial inspection, and many others applications, but for each dataset, the challenge is receiving images by sensors polluted with what we called 'noise' [1]. The noise often corrupts images throughout the acquisition, encoding, compression, transmission, retrieval from storage media, and interfering with natural phenomena, which all can lead to damage data of interest $[2,3]$. There are different types of noise that images can be affected with, such as additive white Gaussian noise, impulse noise, and speckle noise... etc. [4].

The noise has been described as the most critical image problem because it corrupts the image due to blurring, motion, and sensor misfocus, etc..., and is regarded as a major factor in degrading the image quality that leads to image confusion and loss of its characteristics [5]. Therefore, noise reduction is an essential and important technique in digital image analysis and the right first step to be performed before starting any type of investigation like classification, texture analysis, segmentation, feature extraction, ... etc., [6].

Several Image Denoising/ Noise Reduction techniques have been proposed over the last years because it's one of the most common traditional procedures in digital image processing, including wavelet transform and linear filters / non-linear filters as spatial filters to remove or reduce noise from the degraded image while retaining most of the important signal features, but applying these traditional filters like (Mean, Wiener, and Median filters, etc...). It may lead to some problems causing the destruction or loss of vital and essential details in the image, like blurring sharp edges, obliterating /destroying lines and some other fine details existing in the image, as well as failing to eliminate heavy-tailed noise data effectively [7]. Actually, the type and amount of noise present in an image decides the type of filter to be used for denoising a particular noisy image [8].

The spatial domain filtering methods refer to the image operator that aims to remove noise effectively from the digital image by calculating the level gray value of each pixel value $(\mathrm{x}, \mathrm{y})$ depending on the correlation between pixel values/image patches in the original image by using a filter, mask, or window of the same size [9]. Since filtering is a common method of image processing, the term "spatial filtering" refers to filtering operations performed directly on the pixels of a degraded image. The mechanism of the spatial filtering process consists simply of moving the filter mask from the pixel to another pixel value in an image. The filter response at that pixel is computed using a predefined relationship via the filter mask at each pixel value $(x, y)$. The spatial filter can be classified into two primary categories for noise removal: smoothing spatial and sharpening spatial filters. Each of these filters can be either linear or nonlinear in nature [10].

While wavelet transform domain is widely used in the 
signal processing technique, as well as being a superior method for improving image quality because of its multiresolution and subbands properties, in addition to its ability to represent signals in both domains (time and frequency), as is well-known [11]. Donoho and Johnstone proposed wavelet thresholding as a technique for estimating and calculating the value of the signal by employing wavelet transformation capabilities to destroy non-significant coefficients relative to a certain threshold and re-construction the basis signal characteristics based on thresholding coefficients (coefficients subbands) to reduce noise significantly. At the same time, singular information of the original signal can be preserved well, due to its simple calculation and effective influence. There are two major known threshold functions called the hard threshold, which removes the small wavelet coefficients, and the soft threshold function is to shrink large wavelet coefficients. The noise reduction effect of the wavelet threshold function depends on the type of threshold determination such as SureShrink, BayesShrink, Penalized, etc. [12].

Various studies and experiences of researchers have shown that the use of threshold-based wavelet transform domain alone of a deteriorating image is insufficient to achieve an integrated noise-free image, and the resulting image remains blurry. The hybrid algorithm method of wavelet transforms is used in conjunction with a linear/non-linear filter as spatial filters performed on a degraded image to dispose of the greatest value of noise found in the noisy image while retaining the original characteristics by incorporating the advantages of both approaches. WT integrates with spatial filters to resolve the issue of unsharpened edges and poor background quality during the elimination of image blurriness. Simultaneously, the wavelet denoising technique eliminates image details and produces smooth image sharpness. As a result, there is a need for such an algorithm (hybrid method) in image denoising to filtering noise without affecting essential image characteristics, as well as and the elimination of mixed noise and the achievement of an image of appropriate quality with the loss of the smallest possible value of image details during the noise reduction process $[13,14]$.

This review paper presents a variety of noise reduction (or denoising) methodologies based on the wavelet transform and the different spatial filters. It also gives us insights into the methods by which a reliable approach is to be concluded and estimated an approximation of the original image data given its degraded version (noisy image). The rest of this paper is organized as follows. In the "Image denoising problem statement" section, the formulation of the image denoising problem has been presented. While the section "Related Work" explains several significant works in the field of denoising based on digital image denoising techniques with the various types of image noise by related work and summarizes the techniques proposed up to now through the comparative analysis. In the end, the conclusion section gives us a brief of evaluation the literature.

\section{IMAGE DENOISING}

Image denoising aims to recover a high-quality image from its noisy observation (degraded). It's one of the most common problems in image processing. On the other hand, the widespread use of imaging systems makes image restoration significant to the efficiency of the system. Furthermore, the accuracy of the resulting image plays a vital role in user experience and the success of high-level vision tasks, such as object detection and recognition [15]. In general, the problem of image denoising can be modeled mathematically in the following manner:

$$
y=x+n
$$

where:

$y$ : is the observed noisy image (degraded),

$x$ : is referred to the unknown clean image (denoised image),

$n$ : denotes Additive White Gaussian Noise (AWGN) with standard deviation $\sigma_{n}$, which can be calculated in practical applications by different methods, as block-based estimation, principal component analysis-based methods (PCA), and median absolute deviation, etc. $[15,16]$.

The goal of noise reduction is to reduce the noise in original images while minimizing the loss of image features (retaining features) and enhancing the signal-to-noise ratio (SNR). The key obstacles for image denoising are several aspects: Firstly, flat areas should be smooth, edges should be protected without blurring, textures should be preserved, and lastly, should not create new artifacts. Owing to solve the clean image (denoised image) $x$ from the Eq. (1) is an ill-posed problem, we cannot get the efficient solution from the image model with noise [16] To achieve a good image prediction, image denoising has been well studied in the field of image processing over the last few years. Generally, image denoising techniques can be classified mainly as transform domain, spatial domain, or can combine both methods to obtain their advantages $[17,18]$. This paper seeks to highlight this combination in more detail.

\section{RELATED WORK}

Denoising is an image processing technique that seeks to eliminate from digital images different sources of unnecessary noise. Several denoising techniques occur in image processing. The denoising technique in images relies on the noise present type in digital images [19]. Numerous studies have been performed in the domain of digital image denoising using different methods and techniques. The common theme in these works was to choose the appropriate method. Fundamentally, the techniques used to denoising digital images are grouped into two classifications: wavelet transform domain, spatial domain filtering, or combining them, as shown in Figure 1.

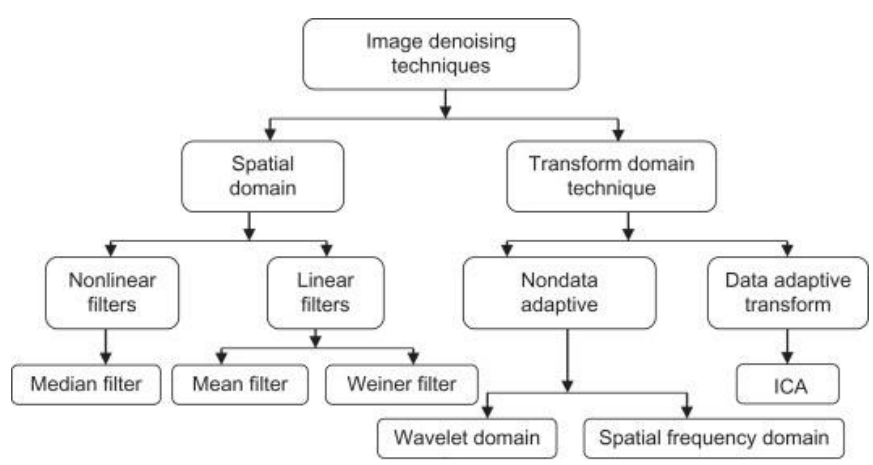

Figure 1. Structural description of different kinds of digital image denoising techniques

In general terms, these techniques aim to remove noise to keep all fine details in the digital image while preserving the 
essential signal features as much as possible $[19,20]$. In this paper, the works will be reviewed according to the following categories: wavelet domain techniques, spatial frequency domain, and wavelet domain and filtering denoising techniques.

\subsection{Wavelet domain technique}

The method includes techniques that rely on wavelet-based noise filtering, which in turn relies on functions. It can be grouped into two key groups, namely: transforms that are nondata adaptive and data-adaptive. Wavelet transform domain techniques are generally described as wavelets, ridgelets, curvelets, and contourlets for digital image denoising. The data-adaptive methods contain an independent component analysis (ICA) transformation technique in which various elements such as projection detection and analysis of factors are covered. While the non-data adaptive transforms method could be classified into two sub-groups, wavelet domain and spatial frequency domain. Spatial methods of filtering are a type of transform domain. It uses the fast-Fourier transform (FFT) and the low-pass filter (LPF). In this case, noise can be removed by discontinuing frequency [21]. While wavelet thresholding is a technique that estimates a signal to reduce the noise by enhancing the capabilities of the wavelet transform. The principal concept of this technique is to eliminate the noise by deleting the insignificant coefficients to a specific threshold and turns it into an efficient and straightforward one. Choosing the threshold depends mainly on the value of the threshold parameter, which leads to an efficient noise reduction $[22,23]$.

There are numerous studies on threshold-based wavelet coefficients. The process of increasing the coefficient efficiency includes removing/deleting the noise of the signal without harming the characteristics regardless of the frequency indication. The procedure includes three steps, the transformation of the linear forward wavelet, the nonlinear thresholding, and finally the transformation of the linear inverse wavelet. These processes are known as denoising by using 2D-Wavelet Transform, including the following main phases, as shown in Figure 2 [23, 24].

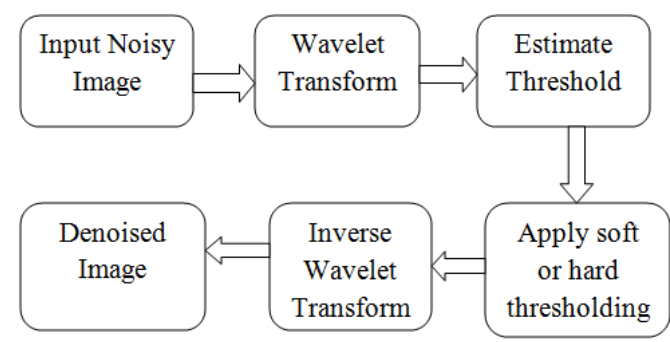

Figure 2. The Block diagram of image denoising using 2Dwavelet transform

(1) The first phase: includes applying the 2D-discrete wavelet transformation to the noisy image (input).

(2) The second phase: includes the threshold calculation, which produces a new clear sub-band by applying each with its related.

(3) The final phase: where 2D-inverse wavelet transformation with the new clear sub-bands re-constructs the new and denoised image (output).

The following studies show the experiments performed in this method:

A new image denoising approach that applies the 2Ddiscrete wavelet transform (2D-DWT) on the noisy image was proposed by Ismael et al., which is the recommended procedure for image analysis as it can split the image to decomposed into four sub-bands, one low-frequency sub-band (LL), the other three high-frequency sub-bands (LH, HL, and $\mathrm{HH}$ ) and function separately on each sub-band frequency. The noise ratio in the noisy image was estimated and measured by a Robust Median Estimator parameter. Furthermore, to assess the performance of the whole process, two measurement parameters were used to calculate the noise in the image, namely (MSE, PSNR). Based on their experimental findings they achieved an improved MSE (mean square error) and PSNR (Peak signal-to-noise ratio) for denoised images by adopting different filters of wavelet transform filters [25].

Three experiments were conducted by Golilarz and Demirel for image denoising in the wavelet domain depending on the soft-threshold function. The first experiment applied the 2Ddiscrete wavelet transform (2D-DWT) along with the soft and hard thresholding values to remove the noise from the degraded image, while the second one used the un-decimated wavelet (UWT) with the hard threshold value on the image. In the last experiment, they used image denoising based on UWT using the soft threshold value. The comparative findings observed the outperforming of the proposed technique in terms of achieving higher PSNR value and improved visual quality while using Lena and Barbara grayscale images (512 x 512 pixels) in and the different contrasts of AWGN noise in addition to retaining most of the image details while eliminating the noise [26].

Koranga et al. focused on wavelet transform using wavelet shrinkage function in their study. Where the wavelet transform has been used to decompose a degraded image to get two groups of image wavelet coefficients, the first one was a wide range of wavelet coefficients that included the features of the image, while the second group of the wavelet coefficients was contained a narrow range of coefficients that included the features of the noise. The soft threshold function (Visu shrink) was then applied to remove some insignificant wavelet coefficients regarding some boundary values. Finally, by reconstruction of inverse decomposition coefficients for each sub-band, the denoised image was obtained. Two measurement parameters were used to calculate the noise in the image, which are (PSNR, RMSE) for various wavelet filters as Db1, Bior1.5, Sym1, and coif1. The results presented an improved performance in eliminating the Gaussian noise from the degraded image and achieved better visual quality [27].

In another scenario investigated by $\mathrm{Xi}$ and $\mathrm{Li}$ [28] introduced an improved denoising algorithm based on a new threshold adjusted by a coefficient considering noise variance. They observed that the current wavelet transform has some limitations and tried to address them by applying the new proposed threshold, for instance, the fixed threshold formula does not follow the changes of the decomposition scale, the hard threshold technique is not continuous at the threshold point, the constant deviation between the original image wavelet coefficients and their estimates always exists in soft threshold technique, and so on. To enhance the denoising effect of the wavelet transform, this paper first layered a new formula for estimating the threshold and a new threshold function calculation method of noisy images with wavelet transform functions. Then, the threshold was calculated with 
the new threshold formula, and the noise was removed through the new threshold function. Finally, re-construct the processed wavelet coefficients to obtain the denoised image. Simulation outcomes showed that the denoising result was better than that of traditional wavelet threshold denoising in terms of (MSE, PSNR) value to calculate the denoised image [28].

In the medical field, Mittal et al. presented a new methodology to improve and remove the high noise of the medical image using the stationary wavelet transform (SWT) method. In their study, an effective and simple technique for adaptive noise removal was used, the SWT 2D denoising method on the medical image that is polluted by noise. The structure-constrained low-rank approximation (SLR) method was applied as a pre-processing to calculate the input images to denoise and recover sharp edges and detail structures. The experimental results demonstrated that the suggested method not only achieved more accurate performance in denoising of medical images in terms of entropy and standard deviation but also improved the image quality, which helped in accurate disease detection [29].

\subsection{Spatial domain filtering}

In the denoising process, filters play a principal role in modifying and enhancing a digital image. There are two major image denoising techniques: spatial domain filtering and wavelet transform domain. Spatial filters that are categorized into non-linear and linear filters are a standard approach for removing noise from degraded image data. These filter types allow low-pass filtering on unit pixel sets, assuming that noise occupies the higher-frequency spectrum region $[19,20]$. In general, spatial filters eliminate noise to a fair degree, but at the cost of blurring images, they make the edges of the images opaque in turn. While non-linear filters as the Median filter remove the noise from the degraded image without any attempts to identify it explicitly. While linear filters as Weiner filter, lead to blur sharp edges, destroy lines and other accurate image details, and perform poorly in the presence of signaldependent noise [30]. The spatial domain processes are indicated as shown in Figure 3.

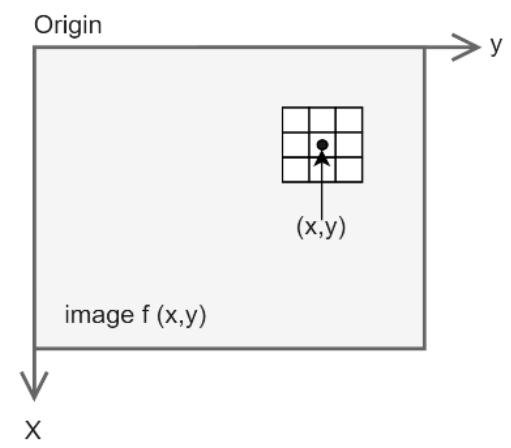

Figure 3. The block diagram of image denoising using spatial domain filtering

Spatial domain processes are denoted by the mathematical expression:

$$
g(x, y)=T[f(x+y)]
$$

where: $g(x, y)$ is processed image, $f(x, y)$ is the input image, and $\mathrm{T}$ is an operator on $f(x, y)$, defined in some a neighborhood of the point $(x, y)$.
While, the rectangular or square (sub-image) area centered at $(x, y)$ is used as a neighborhood around the point $(x, y)$.

Here, $T$ is a gray-level transformation operator of the form $S=T(r)$ where, $r$ and $S$ refer to the gray-levels of $g(x, y)$, and $f(x, y)$ at any point $(x, y)$.

The following studies show the experiments performed in this method:

A new method to image denoising by using the local wiener filter was introduced by Zhang. In his method, he made the use of the advantages of the non-subsampled shearlet transform (NSST), Stein's unbiased risk estimate (SURE), and linear expansion of thresholds (LET) approach in the process of denoising. The procedure involved in his work was first, used NSST in the decomposed noisy image because of the NSST effectiveness as a multi-scale and a multi-directional analysis tool concerning digital image processing. The denoising of high-frequency NSST coefficients was then followed by applying shrinkage functions founded on the local wiener filter. Shrinkage function with the method noise of local wiener filter was adopted efficiently by SURE-LET strategy. In the final step of the procedure, the NSST inverse was made to get the denoised image. Findings made from the method used in the study illustrated that the suggested approach was moreefficient and higher-performing than existing methods such as Shear-Wiener, UWT SURE-LET, and SURE-NeighShrink. At the same time, it presented better results compared with some other wavelet-based approaches [31].

Arazm et al. looked into the improved pixel-wise adaptive wiener filter to eliminate additive white gaussian noise (AWGN) of the scanning electron microscope image (SEM). In their work, the adaptive weight function (AWF) has been discussed in detail, especially for the wiener filter estimation of the local spatial statistics as well as an estimate of the needed noise variance while using the method of linear regression (LR). The suggested filter was eventually compared to the wiener filter and adaptive noise wiener (ANW) to denoise SEM image based on the PSNR value. For various noise variances, the experimental outcomes showed that the suggested filter had improved performance because of the use of adaptive weighted local statistics, which perform better noise removal near sharp edges and a most accurate estimate of noise variance comparison with other mentioned filters [32].

The work of Devi and Sujatha introduced an efficient fingerprint enhancement compared with the existing techniques using the wiener filter. The work was preprocessed in several steps, starting with converting the input fingerprint gray image into the Binary image by applying Otsu thresholding. Then, brighten the binary image using histogram equalization followed by denoising and blurring the equalized binary image and filtering the denoised and blurred image by employing the Wiener filter and ended with enhancing the filtered fingerprint morphological operations through contrast, sharpening, and thinning were performed for more clarity. The image quality was improved and the proposed methodology was effective since it creates a PSNR value that is better in comparison with common methods [33].

Metri and Asha engaged in a study in which a unique image denoising method was proposed. The approach presented in the investigation was performance limits analysis in denoising of images. Through the use of patches that rely on redundancy in patches for image denoising, a wiener filter framework was established. Then, Lena image based on 512X512 resolution was used to examine the proposed algorithm in denoising to evaluate different images that had varying noises. The findings 
illustrate that the various parameters can be estimated through the use of the input of the noisy image. The statistical base of the denoising algorithm is credible and is deliberated in detail. The outcomes indicated that the unique denoising algorithm performed was effective on different images and noise models [34].

A new technique for denoising via a modified adaptive median filter (AMF) was proposed by Zhang et al. The proposed procedure to restoring the degraded image was included in two-phase, first detecting the noise and then eliminating the noise. When detecting the noise in the images, the MSD (minimum set difference) was specified to represent the correlation between pixel-values into the non-pollution group for the determination of high-frequency signal and noise components. In the next phase of the procedure, the noise pixels were replaced with the median value. The findings showed the superiority of the suggested approach compared to the existing techniques as SMF (standard median filter) and AMF in terms of high-noise detection rate, impulse noise reduction effect as well as keep the details right, particularly under the density of high-noise [35].

Tang et al. introduced a new technique for structured light image denoising by the improved (AMF). The goal of the study was to improve an AMF approach to overcome the limitations associated with standard and adaptive median filter (SMF, AMF) approaches employed in reducing structured light images. The AMF used in the study to seeks and achieved better outcomes and involved the following steps. First, they began to detect the actual noise point and local noise density in the noisy image by performing the procedure twice. Second, the filtering window was adjusted in size adaptively based on the density of discovered noise points. Finally, discovered images were filtered from four available directions. The simulation results showed that the suggested technique could effectively preserve the image details while removing noise, as well as achieved better filtering performance compared to SMF and AMF techniques [36].

A new adaptive non-linear filter to remove noise based on the right median filter ( $\mathrm{rm}$ ) was developed by Erkan et al. [37]. A distinct approach was applied to eliminate salt and pepper noise using the adaptive right median filter (ARMF) in their experiment. In the beginning, they defined a unique median method ( $\mathrm{rm}$ - right median) and a distinct adaptive non-linear filter (ARMF) generated through the use of the right median. Then, the ARMF filter results were compared with some known filters by using two image quality metrics (PSNR, SSIM) on the 12 images tests. The results illustrated that ARMF performs better in removing salt and pepper noise than other NAFSMF, MDBUTMF, and DBA methods. However, while it outperforms those other methods at various densities, ARMF failed to achieve that same impressive performance when the noise density is at 80 or 90 percent in the mean percentage. The researchers asserted the variation in performance to be a development linked with the Centre pixel. When the noise density is high, the distance between the regular entries (in the windows that have high-size) and the Centre pixel seems to be large. As such, a drawback is observed in ARMF when the noise densities exceed 80 percent [38].

Lyakhov et al. investigated a practical approach to impulse noise removal using AMF. The best process to eliminate impulse noise is through the median filter, which replaces pixel units for the corrupted image with the similar median values of some neighborhood points. However, the median filter contributes to the image blurring and becomes primarily noticeable while processing images degraded by highintensity impulse noise. In their work, an approach focused on the AMF has been suggested to minimize the negative effect resulting from conventional procedures by adopting iterative image processing with small-masks and pre-processing of the median filter outcomes and improved the efficiency of the processing. The numerical assessment of simulation results by PSNR and SSIM concluded that the suggested approach achieved better clears images from low-intensity and highintensity impulse noise via an intensity of (90-99) percent and it helps solve the impulse noise problem more efficiently [39].

\subsection{Wavelet domain and filtering denoising techniques}

The main differentiation of this technique among the others, like spatial domain filtering technique, that transforms a domain of filtering technique for the given noisy image into another domain, and then it applies the noise reduction procedure on the newly constructed image by following various characteristics of the image and its noise as a second step (Higher coefficients indicates the high-frequency part, i.e., image edges or details, lower coefficients indicate the noise) $[38,40]$. Researchers have done many experiments to enhance denoising efficiency by mixing the wavelet transform filtering domain with the spatial domain filtering methods. The following studies show the experiments performed in this method:

Mridula et al. presented a new algorithm, which combines spatial domain filtering with frequency-domain methods to remove speckle noise form found in synthetic aperture radar images (SAR). The Wiener filter (WF) was used as a preprocessing phase in the spatial domain by the algorithm proposed. Then, by taking the logarithm of the filtered image from the wiener filter, the multiplicative noise model for the speckled image was transformed into an additive one. In the second phase, a decomposition of the wavelet transform was performed with Meyer wavelets, and adaptive soft thresholding was implemented to detail coefficients only ( $\mathrm{LH}$, $\mathrm{HL}, \mathrm{HH})$. Lastly, to obtain a denoised image, the inverse discrete wavelet transformation (IDWT) was calculated by combined the approximation and threshold detail coefficients for the re-constructed image. Then results were quantitatively analyzed by (PSNR) and (SSIM) metrics. Compared to the other algorithm, the simulation outcomes showed that the presented algorithm effectively eliminated speckle noise from the degraded image [41]

A new method to eliminate the noise from computed tomography (CT) images using a combination of locally adaptive wiener filtering with a threshold-based wavelet domain was presented by Diwakar and Kumar. In their proposed methodology, the 2D-discrete wavelet transform (2D-DWT) was performed on the input CT medical image to decompose into low and high-frequency subbands. The detail wavelet coefficient (high-frequency) was adjusted by applying local wiener filtering. The first-intermediate outcome was achieved by implementing the IDWT. As a second step, the first-intermediate was subtracted from the input CT medical image and processed through threshold-based wavelet coefficients for improved edge preservation, thus generating a second-intermediate result. Finally, both-intermediates output was combined to obtain the last outcome of the CT medical image. To estimate and evaluate the performance of the suggested method, the performance metrics (PSNR, SSIM) 
were used. The experimental result of the presented process indicates improved CT medical image with relation to noise suppression and structure conservation [42].

In another scenario, Santhanam and Chithra suggested a hybrid denoising filter to eliminate additive noise in the field of medical images (CT) by combining the absolute difference and Mean filter (ADMF) with a modified median wiener filter (MMWF). The authors focused on reducing the Gaussian noise from computer tomography (CT) degraded images to enhance the medical image quality to increase the ability to diagnose disease by merging the complementary qualities and abilities for each of Median and Wiener spatial filter together with the ADMF filter also. The simulation results showed that the algorithm produces higher (PSNR) and lower (MSE) values. The average (MSE) values for new hybrid filtering techniques compared with similar approaches (TDBF, DWTTV, EPHF, MMWF, and ADMF) have been calculated. The new hybrid method showed a decrease of $73.76 \%$ MSE value and an increase of $26.24 \%$ concerning the PSNR value. The indicated outcomes that the suggested approach in their study was more-suitable for eliminating Gaussian noise in the field of medical images (CT) than other algorithms [43].

A new technique to eliminate image noise by adopting a median filter (MF) in the wavelet domain was suggested and tested by Ramadhan et al. They examined various types of wavelet transformation filters by coupling with a median filter to test the proposed approach and achieve better results for the process of image noise removing. After the test has been done, a suitable filter was chosen for their work. The algorithm showed that the quality of the image produced through the technique suggested of corrupted images involving Gaussian noise compared to the PSNR value achieves better results than traditional methods for wavelet-based thresholding and Median filter each alone. The experimental results appeared the higher levels of PSNR for the denoised images, providing clear images and improve the visual quality [44].

A research was suggested by Longkumer and Gupta about a new technique that used wavelet transform on the input noised image with thresholding function and Weiner Filter. In their proposed method, an investigation into several research papers on image denoising was done and their performance analyzed. A new approach based on the Daubechies wavelet family (db4) was applied which uses a Weiner filter with approximation coefficients (LL) sub-band, and soft thresholding with the detail coefficients (HH, HL, LH) subbands after adding Gaussian, salt and pepper noise to the image. Then re-constructed the denoised image by inverse wavelet transform. The performance of the suggested algorithm assessed in terms of measurement criteria (MSE, PSNR), competitive performance compared to existing techniques has been observed in removing noise for images contaminated with AWGN noise and salt and pepper noise. The method showed the effectiveness of the proposed algorithm [45].

Aghabalaei et al. suggested a new procedure that combines a Kalman filter with the wavelet transformation to eliminate speckle-noise from synthetic aperture radar (SAR) images. In their study, the dataset was collected from different paths and periods during their capture. Therefore, the single look complex (SLC) data was initially then co-registered into the image of the first path, and then a re-sampling of the images was performed process on a similar scale in both directions (range, azimuth). The first image was de-speckled as the second step by applying the B-spline wavelet combined with the Birgé-Massart strategy to obtain denoising thresholds. Lastly, to get the de-speckled time-series (SAR) image, the recursive Kalman filter was used on other images. They used each of the quantitative and qualitative tests, including the two criteria (ENL, EEI) for evaluation. The performance results of the suggested method were better in terms of preserving the major edge structures and details compared to other current filters such as Lee-Sigma and Frost filters in eliminating speckle-noise from (SAR) images. Furthermore, it is computationally effective in maintaining spatial accuracy and reducing remote sensing processing [46].

As regards the objective and subjective performance of the conventional filtering, a combination algorithm applied by Choi and Jeong showed a better performance to eliminate speckle noise of SAR image and retaining edge details by applying speckle-reducing anisotropic diffusion filter (SRAD) with the threshold-based wavelet transform and guided filter. The SRAD filter was adopted as a pre-processing filter to retain useful information and enhance the SAR image quality. A logarithmic transformation (log) function was then used to further eliminate the multiplicative noise residual in the prefiltered image by transforming speckle noise to additive one. As a penultimate step, the resulting image was decomposed to a multi-resolution process by a soft thresholdbased discrete wavelet transform (DWT) and guided filter for the four decomposed subband images (LL, LH, HL, HH). Finally, the inverse wavelet transformation (IDWT) and an exponential function were implemented to the re-constructed image to get a denoised image [47].

Dass suggested a new approach to speckle-noise reduction of ultrasound images by applying bacterial foraging optimization (BFO) cascaded with wavelet transformation and Wiener filter. In his technique, the Wiener filter was adopted as a pre-processing filter to eliminate speckle-noise from the degraded image. In order to further eliminate the noise residual in the prefiltered image, a wavelet packet was used with the adaptive soft thresholding function to detect and remove noise from the affected pixels. Then inverse wavelet transformation (IDWT) and an exponential transform function were implemented to reconstruct the prefiltered image to get a denoised image. Lastly, the BFO algorithm reduces the error percentage between both the speckled-image and the despeckled output-image of the homomorphic framework after pre-processing, maintaining the finer details, as well as the error percentage. The technique presented superior results compared to other techniques based on two measurement criteria (MAE, PSNR). The results displayed that the merger of DWT with Wiener filter performs better outcomes in preserving the image by eliminating noise from images. Simultaneously, the combined BFO and AMF achieve improvement in the visual and statistical results [48].

Moving to the medical images field, Tayade and Bhosale presented a modified technique that works on improving the low-resolution (LR) medical image by using the dual-tree complex-based wavelet transform (DTCWT) along with image interpolation and wiener filter. In their procedure, the cascaded structure of DTCWT was employed to produce various frequency bands for the evaluation and analysis of their algorithm due to the properties of shift-invariance and good directional selectivity. While the denoising approach of their proposed has been based on the Wiener filter and dualtree complex. The LR of the input image was decomposed into multiple frequency sub-bands by using six-level of decomposition in order to the production of various frequency 
sub-bands for analysis. Simultaneously, numerous quality standard criteria such as PSNR, MSE, and SSIM were employed to assess the technique of resolution enhancement and quality of the denoised medical image. The findings indicated that the suggested process has a better smoothness and higher accuracy balance of image compared to the discrete wavelet transform (DWT) as well as less redundant than the stationary wavelet transform (SWT) [49].

Researchers were also interested in enhancing computed tomography (CT) Luo et al. investigated a new denoising algorithm of CT images by applying a double-density dualtree complex based-wavelet transform (DDCWT) with a modified thresholding technique. The method starts with decomposing the noisy CT medical image into low and highfrequency wavelet coefficients, then adopting a modified threshold function to enhance the performance and preserve effective wavelet coefficients for all sub-bands. The thresholding value was determined depended on the modified threshold function. In the end, the denoised image was obtained by re-constructing inverse decomposition coefficients for each sub-band (low and high-frequency) of DDCWT. The evaluation of the method included two measurement parameters computed visual quality between the input image and the out image, namely (MSE, PSNR). The experimental results indicated that the denoising image algorithm based on DDCWT performs better and maintains more rich details in CT images than the classical DTCWT algorithm [50].

Qian proposed a new algorithm that combined the improved threshold-based wavelet decomposition with the median filter to degraded image denoising. In his research, the improved threshold function has been employed on wavelet detail coefficients (high-frequency) mixed by noise which was denoised of each level after two-level decomposition of wavelet was performed on the noisy image, then reconstructed the restored image by wavelet approximation, and the denoised wavelet details of each level. Eventually, the Median filtering was twice performed for the re-constructed images to obtain the denoised image. The findings of the new method showed that the denoising effect of the improved threshold technique was better from soft and hard thresholding techniques in eliminating Gaussian noise and Median filter in eliminating salt and pepper noise based on measurement parameters (PSNR). Therefore, the presented algorithm can effectively eliminate the multiple random noises into the degraded image and be implemented in engineering image field denoising because of strong adaptability and a morestable noise reduction effect [51].

A year after, Qian presented another combined algorithm for image denoising that consists of AMF with an improved threshold-based wavelet transform (WT). In the first step, the upper limit of the template was set in his algorithm, the salt and pepper noise was removed by employed AMF filter within the limit. In the second step, a two-level decomposition of wavelet was performed on the degraded image. The coefficients of high-frequency were denoised by applying an improved threshold technique, then re-constructed the restored image by coefficients of low-frequency. MATLAB simulation outcomes showed that the investigated approach (AMF-WT) was superior to the AMF filter, reducing salt and pepper noise. The improved threshold function development had a better denoising effect compared to the soft and hard threshold functions [52].

Nishu et al. suggested combining the speckle reducing anisotropic diffusion filter (SRAD) with Bayesian thresholdbased wavelet transform to eliminate speckle noise effectively and enhance image visual quality. In their work, the uncompressed noisy image has been processed by applying an SRAD filter, and then the logarithmic transformation converted the speckle noise into additive noise. Finally, the DWT employing a soft thresholding function (Bayesian) has been done on the log compressed image to get a denoised image. The results showed that the performance of the suggested technique, compared to several other different techniques, provides an improved visual quality based on the measurement parameters like RMSE, PSNR, SSIM, and computational time (s) metrics [53].

Fan et al. investigated two different methods to remove Gaussian white noise from the image with a threshold-based wavelet and Wiener filter. In the first method, the DWT was made on a grayscale image to decomposed into approximation and detail coefficients. Then wavelet coefficients were adjusted using wavelet thresholding by eliminating the noisyaffected coefficients from the image. While in the second method, the image was analyzed using a wiener filter in the wavelet domain. Finally, after noisy coefficients are removed from both methods (first, second), the denoised image was reconstructed by inverse DWT. By comparing the performance of both methods, they observed that the second method (wiener filter in the wavelet domain) was better to remove Gaussian noise from the image [54].

A new procedure for image denoising affected by additive white gaussian noise (AWGN) using threshold-based discrete wavelet transformation with wiener filter by Ferzo and Mustafa was adopted. They employed multi-resolution wavelet transform to reduce the noise from pixel units of the damaged image by relying on four thresholding functions into all wavelet filters. Then, the inverse discrete wavelet transform (2D-IDWT) was implemented to the re-constructed image to get a denoised image. Simultaneously, the Wiener filter was used before/after also to remove noise and complete the denoising procedure. The Peak Signal to Noise Ratio (PSNR) was chosen to measure the performance efficiency that led to an improvement of about 17.5 percent compared to other existing work, as well as to the improved quality and edge preservation of the image [55].

Table 1 below provides a summary of the selected researches that deals with image denoising techniques of the noisy image in most fields by using multi-resolution wavelet transform in conjunction with spatial domain filters for the previous five years through a comprehensive analysis of various sources and technical principles that included denoising filtering techniques, pros and cons, measurement parameters, types of noise used, as well as wavelet decomposition levels. 
Table 1. The summary of the selected researches that deal with digital image denoising by using multiresolution wavelet domain and spatial domain filter

\begin{tabular}{|c|c|c|c|c|c|c|}
\hline Ref. & $\begin{array}{c}\text { Denoising Filtering } \\
\text { Techniques }\end{array}$ & Pros & Cons & Noise Type & $\begin{array}{c}\text { Measurement } \\
\text { Parameters } \\
\text { Used }\end{array}$ & $\begin{array}{c}\text { Wavelet } \\
\text { Decomposition } \\
\text { Levels }\end{array}$ \\
\hline
\end{tabular}

[25] Discrete Wavelet Transform (DWT)

Un-decimated

[26] Wavelet Transform (UWT)

[27] Visu threshold-based Wavelet Transform

[28]

Improved Wavelet Threshold Transform

[31] Local Wiener Filter

\section{Adaptive Wiener} Filter

\section{Wiener Filter}

$$
\begin{gathered}
\text { Modified Adaptive } \\
\text { Median Filtering }
\end{gathered}
$$

[36] Improved Adaptive Median Filtering
Able to transform the digital image into sub-bands and operate on each frequency individually

A better denoising capability and achieve smoothness and accuracy due to do not downsample the approximation and detail coefficients at each decomposition

Adopts different wavelet families with the Visu thresholding technique for decomposition and denoising

It has good localization and multiresolution characteristics and effectively solves the

defect that the approximation degree between the clean image and re-constructed image

Removes noise from medical images quickly and accurately while enhancing visibility

Capable of analyzing images in multi-scale, multi-direction and shift-invariant

Able to deals with different noise variances

Removes different forms of noise from fingerprint images characteristically and effectively

Effective on various models of images and noise

Capable of detecting high noise rate, eliminate the impulse noise effectively and keep the image details well

Able to assess the actual noise point as well as local noise density

Able to adapt and expand the window size
Deals with a

specific amount of original images

standard deviation

Uses only the soft thresholding technique

Covers the cameraman image with noise variance $\sigma=$ 10 only

The digital image domain and noise variance used has not been specified

Covers medical images as well as it uses only the Haar wavelet

Rely only on PSNRs to assess the performance of the approach against existing techniques

Ineffective with larger masks because it leads to image blurring

DB2 was used only

Only deals with grayscale images and the process is time-consuming

To keep the image details better, the window size should be smaller

Deals with one form and a small ratio of noise

Effective in removing salt and pepper noise only
Gaussian

Noise

MSE, PSNR

Two-level

\section{AWGN}

Noise

PSNR

One-level

\section{Gaussian}

Noise

PSNR, RMSE

Two-level

Non-specific

MSE, PSNR

Non-specific

$\begin{array}{cc}\text { Adaptive } & \text { Entropy and } \\ \text { Noise } & \text { Standard } \\ & \text { Deviation }\end{array}$

Two-level

Gaussian

White Noise

PSNR

Spatial domain (one-level)

\section{AWGN}

Noise

PSNR

Spatial domain (one-level)

Gaussian, speckle and
Salt and pepper

Noise

Poisson,

AWGN and

Salt and

pepper

Noise

Impulse

Noise

PSNR, SSIM

Spatial domain (one-level)

Structured

Light

NMSE, PSNR

Spatial domain (one-level)

Salt and

Pepper

SSIM, PSNR

Noise

Spatial domain (one-level)

Spatial domain (one-level) (ARMF) 


\begin{tabular}{cc}
\hline Ref. & $\begin{array}{c}\text { Denoising Filtering } \\
\text { Techniques }\end{array}$ \\
\hline$[39]$ & $\begin{array}{c}\text { New Adaptive } \\
\text { Median Filtering }\end{array}$ \\
{$[41] \quad \begin{array}{c}\text { Wiener Filtering and } \\
\text { Adaptive Wavelet } \\
\text { Thresholding }\end{array}$} \\
& Threshold-based-
\end{tabular}

[42] Threshold-basedWavelet Transform with Wiener Filter

[43] Absolute Difference and Mean (ADMF) and Modified Median Wiener filter (MMWF)

[44] Median Filter (MF) in the Wavelet Domain

[45] Discrete Wavelet Transform using Soft Thresholding and Weiner Filter

[46] Kalman Filter with Wavelet Transform

[47] Speckle-reducing anisotropic diffusion filter (SRAD) with the threshold-based wavelet transform and guided filter

[48] Bacterial Foraging Optimization (BFO) Cascaded with Wavelet Transform and Wiener Filter

[49] dual-tree complexbased wavelet transform (DTCWT) along with image interpolation and wiener filter

[50] double-density dualtree complex basedwavelet transform (DDCWT) with a modified thresholding technique

[51] Improved Thresholdbased Wavelet Transform and Median Filter
Removes both low-intensity

and high-intensity noise with efficiency

Removes speckle noise effectively and significantly

Provide significant potential for edge extraction to improve the quality of denoised CT medical images concerning noise suppression and structure preservation

Remove Gaussian noise from CT images effectively and significantly

Able to retrieve more details from the original image, provision of a clear image and better visual quality

Effectively denoising and compression while preserve edges and details

Preserves the main edge structure and the spatial resolution while reduce the process time

Removes noise from (SAR) image Effectively, avoids edge details loss and significant features

Able to retrieve most of the fine details and preserve the edges of ultrasound images

Improve the accuracy of the low-resolution medical image

Able to decompose with significantly higher dimensions in direction selectivity, as well as provide approximation shiftinvariance

Removes mixed noise effectively, has strong adaptability, as well as deals with fingerprint images and printed circuit boards (PCB) in the complex-noise environment

\begin{tabular}{|c|c|c|c|}
\hline Cons & Noise Type & $\begin{array}{c}\text { Measurement } \\
\text { Parameters } \\
\text { Used }\end{array}$ & $\begin{array}{c}\text { Wavelet } \\
\text { Decomposition } \\
\text { Levels }\end{array}$ \\
\hline $\begin{array}{l}\text { Covers the } \\
\text { grayscale Lena } \\
\text { image only }\end{array}$ & $\begin{array}{l}\text { Impulse } \\
\text { Noise }\end{array}$ & PSNR, SSIM & $\begin{array}{l}\text { Spatial domain } \\
\quad \text { (one-level) }\end{array}$ \\
\hline $\begin{array}{l}\text { Covers synthetic } \\
\text { aperture radar } \\
\text { (SAR) field only }\end{array}$ & $\begin{array}{l}\text { Speckle } \\
\text { Noise }\end{array}$ & PSNR, SSIM & Two-level \\
\hline $\begin{array}{l}\text { Unable to retrieve } \\
\text { all of the re- } \\
\text { constructed CT } \\
\text { images }\end{array}$ & $\begin{array}{l}\text { Gaussian } \\
\text { Noise }\end{array}$ & PSNR, SSIM & One-level \\
\hline $\begin{array}{l}\text { Covers medical } \\
\text { image affected by } \\
\text { Gaussian noise only }\end{array}$ & $\begin{array}{l}\text { Gaussian } \\
\text { Noise }\end{array}$ & PSNR, MSE & $\begin{array}{l}\text { Spatial domain } \\
\text { (one-level) }\end{array}$ \\
\hline $\begin{array}{l}\text { Effective in } \\
\text { removing one type } \\
\text { of noise }\end{array}$ & $\begin{array}{c}\text { Additive } \\
\text { Gaussian } \\
\text { Noise }\end{array}$ & PSNR & One-level \\
\hline $\begin{array}{l}\text { Denoising image in } \\
\text { detail coefficients } \\
\text { was adopted on the } \\
\text { db4 wavelet only }\end{array}$ & $\begin{array}{l}\text { Gaussian, } \\
\text { and Salt } \\
\text { and Pepper } \\
\text { Noise }\end{array}$ & PSNR, MSE & One-level \\
\hline $\begin{array}{l}\text { Covers synthetic } \\
\text { aperture radar } \\
\text { (SAR) image } \\
\text { systems field only }\end{array}$ & $\begin{array}{l}\text { Speckle } \\
\text { Noise }\end{array}$ & ENL, EEI & Five-level \\
\hline $\begin{array}{l}\text { Process synthetic } \\
\text { aperture radar }\end{array}$ & $\begin{array}{l}\text { Speckle } \\
\text { Noise }\end{array}$ & $\begin{array}{l}\text { RMSE, PSNR, } \\
\text { SSIM and ENL }\end{array}$ & One-level \\
\hline
\end{tabular}

aperture radar

(SAR) imaging

systems only

Covers medical

Speckle

PSNR, MAE

Non-specific only

Covers medical images only.

The process has complications and time-consuming

Complex and timeconsuming process

PSNR, MSE

Non-specific

Six-level ultrasound images

Denoising image in

Two-level sym5

wavelet coefficients and SSIM

PSNR
Non-specific
Salt and
Pepper,
Gaussian,
Speckle
Noise
Noise
Non-specific




\begin{tabular}{|c|c|c|c|c|c|c|}
\hline Ref. & $\begin{array}{c}\text { Denoising Filtering } \\
\text { Techniques }\end{array}$ & Pros & Cons & Noise Type & $\begin{array}{l}\text { Measurement } \\
\text { Parameters } \\
\text { Used }\end{array}$ & $\begin{array}{c}\text { Wavelet } \\
\text { Decomposition } \\
\text { Levels }\end{array}$ \\
\hline [52] & $\begin{array}{l}\text { Adaptive Median } \\
\text { Filtering with } \\
\text { Threshold- based } \\
\text { Wavelet Transform } \\
\text { (AMF-WT) }\end{array}$ & $\begin{array}{l}\text { Able to adjust the size of the } \\
\text { template according to the } \\
\text { noise concentration }\end{array}$ & $\begin{array}{l}\text { Complex algorithm } \\
\text { and needs prior } \\
\text { knowledge of noise } \\
\text { concentration }\end{array}$ & $\begin{array}{l}\text { Salt and } \\
\text { pepper } \\
\text { Noise }\end{array}$ & PSNR & Two-level \\
\hline [53] & $\begin{array}{l}\text { Speckle Reducing } \\
\text { Anisotropic Diffusion } \\
\text { filter (SRAD) in } \\
\text { combination with the } \\
\text { Bayesian Threshold- } \\
\text { based Wavelet } \\
\text { Transform }\end{array}$ & $\begin{array}{l}\text { Eliminates a difficult type of } \\
\text { noise, and provides improved } \\
\text { visual quality of the re- } \\
\text { constructed image by } \\
\text { reaching a good compromise } \\
\text { in the speckle-noise reduction } \\
\text { and edges preservation }\end{array}$ & $\begin{array}{l}\text { The process takes a } \\
\text { long time due to the } \\
\text { combination of } \\
\text { more than one } \\
\text { algorithm }\end{array}$ & $\begin{array}{l}\text { Speckle } \\
\text { Noise }\end{array}$ & $\begin{array}{l}\text { PSNR, RMSE } \\
\text { and SSIM }\end{array}$ & Two-level \\
\hline [54] & $\begin{array}{l}\text { Threshold-based } \\
\text { Wavelet Transform } \\
\text { and Wiener Filter }\end{array}$ & $\begin{array}{l}\text { Use the improved soft } \\
\text { thresholding to improve the } \\
\text { denoising effect } \\
\text { and to improve the } \\
\text { performance of both } \\
\text { thresholding (soft and hard) }\end{array}$ & $\begin{array}{l}\text { Ineffective in } \\
\text { improving } \\
\text { performance along } \\
\text { the edges }\end{array}$ & $\begin{array}{c}\text { Gaussian } \\
\text { White Noise }\end{array}$ & SNR & Non-specific \\
\hline [55] & $\begin{array}{c}\text { Threshold-based } \\
\text { Discrete Wavelet } \\
\text { Transform (DWT) } \\
\text { with Wiener Filter }\end{array}$ & $\begin{array}{l}\text { Use a combination of the } \\
\text { threshold-based wavelet } \\
\text { domain with the Wiener filter } \\
\text { by applying many different } \\
\text { threshold functions in each } \\
\text { case }\end{array}$ & $\begin{array}{c}\text { More time needed } \\
\text { for the process } \\
\text { because more than } \\
\text { one combination } \\
\text { and several cases } \\
\text { existed }\end{array}$ & $\begin{array}{l}\text { Additive } \\
\text { White } \\
\text { Gaussian } \\
\text { Noise }\end{array}$ & PSNR & Three-level \\
\hline
\end{tabular}

After assessing and analyzing the methods used in all previous studies depending on Table 1, which provides a comprehensive summary of this evaluation, it turns out that many variables, such as threshold function, the type of noise and filter, and the field of the image used, all play a critical role in obtaining images with entirely reduced noise. To evaluate the efficiency of the proposed methods, all of these characteristics must be considered. Following a study and evaluation of the proposed and implemented working methods covered by this research, the following conclusions were reached:

(1) Several studies focused on the medical imaging field, such as Computed Tomography Scan (CT), X-Ray image, Ultrasound image, and other research has also covered Earth science aspects, such as Synthetic Aperture Radar Image (SAR). Whereas other techniques concentrated on the standard images like Lena, Cameraman, and Barbara, etc...). All these studies aimed to eliminate noise from the deteriorating (confusing) image relying on the domain in which the image has been used.

(2) According to the covered works in this research, various Metric/measurement parameters such as (MSE, PSNR, SSIM, RMSE, ENL, EEI, and so on...) are computed to analyze and assess techniques based on the value of denoising image compared to the degraded image (noisy), the PSNR was the most suitable and convenient criteria that can be used to indicate the improvement ratio for the denoising applied methods.

(3) As per our study and review of previous works, spatial filters also have the ability to give an improvement in the results of applying the image denoising methods as well as, the noise in the digitally degraded image can be filtered out while preserving the desirable components by using different types of spatial filters. As a result, the choice of the filter plays a significant role in denoising techniques. Actually, the form and volume of noise present in an image determine the type of filter to be used for denoising a particular noisy image since different filters are capable of efficiently removing different forms of noise, for example, Wiener Filter effective on various models of images and noise and capable of analyzing images in multi-scale, multi-directional, and shift-invariant formats. While the Median filter is capable of detecting high noise rate, reduce the impulse noise effectively, and keeps the details of the image well. Table 2 summarizes details about spatial domain filters and the noises that they can suppress:

Table 2. The filter's name and properties

\begin{tabular}{|c|c|c|}
\hline Filter name & Filter type & $\begin{array}{l}\text { Noise type and } \\
\text { performance }\end{array}$ \\
\hline Mean filter & $\begin{array}{c}\text { linear } \\
\text { smoothing }\end{array}$ & Gaussian noise \\
\hline Weiner filter & $\begin{array}{l}\text { linear } \\
\text { smoothing }\end{array}$ & Additive noise \\
\hline Gaussian filter & $\begin{array}{l}\text { linear } \\
\text { smoothing }\end{array}$ & Gaussian noise \\
\hline Median filter & $\begin{array}{l}\text { Non-linear } \\
\text { smoothing }\end{array}$ & Impulsive noise \\
\hline $\begin{array}{l}\text { Max and Min } \\
\text { filter }\end{array}$ & $\begin{array}{l}\text { Non-linear } \\
\text { smoothing }\end{array}$ & Salt and Pepper noise \\
\hline Midpoint filter & $\begin{array}{l}\text { Non-linear } \\
\text { smoothing }\end{array}$ & $\begin{array}{c}\text { Gaussian and uniform } \\
\text { noise }\end{array}$ \\
\hline $\begin{array}{l}\text { Rank order EV } \\
\text { filter }\end{array}$ & $\begin{array}{l}\text { Non-linear } \\
\text { smoothing }\end{array}$ & White noise \\
\hline $\begin{array}{l}\text { Rank order ER } \\
\text { filter }\end{array}$ & $\begin{array}{l}\text { Non-linear } \\
\text { smoothing }\end{array}$ & $\begin{array}{c}\text { Complicated noise with } \\
\text { impulsive component }\end{array}$ \\
\hline $\begin{array}{l}\text { Rank order } \\
\text { KNV filter }\end{array}$ & $\begin{array}{l}\text { Non-linear } \\
\text { smoothing }\end{array}$ & $\begin{array}{l}\text { White noise and Speckle } \\
\text { noise }\end{array}$ \\
\hline Laplacian filter & $\begin{array}{c}\text { Linear } \\
\text { sharpening }\end{array}$ & Edge detection \\
\hline Gradient filter & $\begin{array}{l}\text { Non-Linear } \\
\text { sharpening }\end{array}$ & Noise reduction \\
\hline
\end{tabular}


(4) Many studies have focused on applying 2DWT as a powerful tool for signal and image processing with the aim of eliminating noise from the damaged image in various fields, especially when it can be used as a multi-resolution transformation. The multi-resolution plays a critical role in increasing denoising improvement. However, it has some shortcomings like shift variance property due to the downsampling process and lack of directional selectivity.

(5) According to our study and review of previous work, the Un-decimated Wavelet Transform (UWT), give extra improvement with respect to the Wavelet Transform (WT) from the point of view of the ratio of the noise removed because this kind will deal with all the pixels without any down-sampling process which means increasing the amount of image information. But, from the process time side, this method will spend more time.

(6) Our study and analysis of previous works also have led us to conclude that the threshold technic shows a critical role inside the image denoising based on using the wavelet transformation. The threshold function selection is a significant point that will reflect on the results of the denoising system.

(7) Several techniques have developed a simple and effective method to eliminate noise concerning the processing time in order to achieve a noise-free image. In contrast, others have developed complex methods such as DTDWT, DTCWT, and SRAD to obtain noise-reduced images, better visual quality, and achieve an effective result. However, there is still a lack of image clarity, and also it takes a long time to process.

(8) As per our review of previous works, some studies were observed to have focused on the use of the convolution masks and the moving window model of the noisy image (template size) to denoising the image through the correlation between pixel values/image patches in the original image by using a filter, mask, or window of the same size. Simultaneously, others concentrate on the details of the sharp edges and vital characteristics of the noisy image.

(9) Since there are many forms of noise that occur concurrently within the image at times, so several works have been based on the noisy image that includes mixed noise in order to minimize the mixed noise that exists in the image, as a result, the denoising image across one form of filter is sometimes ineffective in noise removal, while a combined hybrid denoising approach incorporating the merged of several filters is required to be as efficient as possible at removing the mixed noisy image.

(10) Finally, our analysis and evaluation of previous research have concluded that using the hybrid method by mix the 2DWT image denoising method with the use of the spatial filter can be considered an effective idea to get the benefits of both sides and to increase the improvement. The advantage of using the hybrid method is to provide significant potential for edge extraction to improve the quality of denoised images concerning noise elimination and preservation of structures and image characteristics.

\section{CONCLUSIONS}

As the complexity and requirements of available image denoising have grown, research in this field has become increasingly important. In this research, the main issues of image degradation and restoration have been reviewed for the previous studies with several related works on the mechanisms and different methodologies that applied to get the optimum of removing noise from degraded images associated with the use of spatial filters and the mechanisms of process based on implementing denoising in the wavelet domain. The survey of the above studies on various types of noise removal techniques revealed that some of them depend on the use of only WT domain such as (DWT, SWT, etc...), while others dependent on complex WT domain such as (DTDWT, DTCWT, and SRAD) using filters for a single or mixed form of noise including (Gaussian, salt \& pepper, Poisson, and speckle noise, etc...) in the deteriorating image to obtain the noise reduction image as close as possible to the original image depending on the hard and soft threshold functions. The results provided strong indicators that the hybrid method process by combining the denoising technique in the spatial domain filters with the WT domain based on threshold function produces improved results compared to either method alone. A variety of traditional and novel measurement parameters has been adopted to evaluate the performance of the various methods used. However, these methods still lack behind in the better visual quality of the image. Additional can be determined performance parameters to investigate the behavior of hybrid methods that achieve a successful balance of noise reduction in various noise reduction fields.

Finally, after assessing and analyzing all the methods used in this research, the work presented will provide essential material for researchers and a reference, especially new entrants in the field of image denoising, because the research touched on all the techniques and mechanisms used to restore the digital image affected by noise in most fields. Besides, knowledge of the pros and shortcomings trend for each method and the type of algorithm that is most appropriate for each form of noise found in the image via an analysis of various technical sources and principles. In future work, we plan to investigate how to deal with other forms of noise, especially those existing in real-life. Furthermore, the image denoising technique can also be developed and expanded to other applications.

\section{REFERENCES}

[1] Raghuwanshi, N., Chourasia, B. (2019). A study on different type of noise and noise filtering method. Journal of Engineering, Computing and Architecture, 9(12): 3037.

[2] Alisha, P.B., Sheela, K.G. (2016). Image denoising techniques-an overview. IOSR J. Electr. Commun. Eng.

[3] Win, N.N., Kyaw, K.K.K., Win, T.Z., Aung, P.P. (2019). Image Noise Reduction Using Linear and Nonlinear Filtering Techniques. International Journal of Scientific and Research Publications (IJSRP), 9(8): 92113.

[4] Mehendale, T., Ramina, V., Pinge, S., Kulkarni, S. (2020). Analysis of the Effects of Different types of Noises and Wavelets used in Denoising of an Image using Wavelet Transform. In 2020 11th International Conference on Computing, Communication and Networking Technologies (ICCCNT), pp. 1-5. https://doi.org/10.1109/ICCCNT49239.2020.9225629

[5] Charmouti, B., Junoh, A.K., Mashor, M.Y., Ghazali, N., Wahab, M.A., Muhammad, W.Z.A.W., Beroual, A. (2019). An overview of the fundamental approaches that yield several image denoising techniques. Telkomnika, 17(6). 
https://doi.org/10.12928/TELKOMNIKA.v17i6.11301

[6] Singh, L., Janghel, R. (2019). Image denoising techniques: A brief survey. Harmony Search and Nature Inspired Optimization Algorithms, 731-740. https://doi.org/10.1007/978-981-13-0761-4_70

[7] Song, Q., Ma, L., Cao, J., Han, X. (2015). Image denoising based on mean filter and wavelet transform. In 2015 4th International Conference on Advanced Information Technology and Sensor Application (AITS), pp. 39-42. https://doi.org/10.1109/AITS.2015.17

[8] Dwivedy, P., Potnis, A., Soofi, S., Giri, P. (2017). Performance comparison of various filters for removing different image noises. In 2017 International Conference on Recent Innovations in Signal processing and Embedded Systems (RISE), pp. 181-186. https://doi.org/10.1109/RISE.2017.8378150

[9] Ansari, I.R. (2013). Image denoising using spatial domain filters. International Journal of Advanced Technology in Engineering and Science (IJATES), 1(2): 42-53.

[10] Shenbagavadivu, S., Devi, M.R. (2013). An investigation of noise removing techniques used in spatial domain image processing. International Journal of Computer Science and Mobile Computing, 2(7): 378-384.

[11] Wang, G., Wang, Z., Liu, J. (2017). A new image denoising method based on adaptive multiscale morphological edge detection. Mathematical Problems in Engineering, 2017: 1-11. https://doi.org/10.1155/2017/4065306

[12] Lin, L. (2018). An effective denoising method for images contaminated with mixed noise based on adaptive median filtering and wavelet threshold denoising. Journal of Information Processing Systems, 14(2). https://doi.org/10.3745/JIPS.02.0083

[13] Kethwas, A., Jharia, B. (2015). Image de-noising using fuzzy and wiener filter in wavelet domain. In 2015 IEEE International Conference on Electrical, Computer and Communication Technologies (ICECCT), pp. 1-5. https://doi.org/10.1109/ICECCT.2015.7226071

[14] Kumar, P., Agarwal, S.K. (2015). A color image denoising by hybrid filter for mixed noise. International Journal of Current Engineering and Technology (IJCET), 5 .

[15] Gu, S., Timofte, R. (2019). A brief review of image denoising algorithms and beyond. Inpainting and Denoising Challenges, 1-21. https://doi.org/10.1007/978-3-030-25614-2_1

[16] Fan, L., Zhang, F., Fan, H., Zhang, C. (2019). Brief review of image denoising techniques. Visual Computing for Industry, Biomedicine, and Art, 2(1): 112. https://doi.org/10.1186/s42492-019-0016-7

[17] Jakhete, M.D. (2015). Classification of Image Denoising Techniques.

[18] Pravina, L.S., Shunmugan, S. (2020). Survey on image denoising methods. In 2020 Fourth International Conference on I-SMAC (IoT in Social, Mobile, Analytics and Cloud) (I-SMAC), pp. 411-415.

[19] Koundal, D., Kadyan, V., Dutta, P., Anand, V., Aggarwal, S., Gupta, S. (2020). Computational techniques in biomedical image analysis: Overview. Advances in Computational Techniques for Biomedical Image Analysis, 3-31. https://doi.org/10.1016/B978-0-12820024-7.00001-3

[20] Swamy, S., Kulkarni, P.K. (2020). A Basic Overview on
Image Denoising Techniques. International Research Journal of Engineering and Technology, 7(5): 850-857.

[21] Jain, P., Tyagi, V. (2016). A survey of edge-preserving image denoising methods. Information Systems Frontiers, $18(1)$ : 159-170. https://doi.org/10.1007/s10796-014-9527-0

[22] Sunkara, J.K. (2019). Selection of wavelet basis function for image compression-a review. ELCVIA Electronic Letters on Computer Vision and Image Analysis, 18(1): $1-20$.

[23] Guhathakurta, R. (2017). Denoising of image: A wavelet based approach. In 2017 8th Annual Industrial Automation and Electromechanical Engineering Conference (IEMECON), pp. 194-197. https://doi.org/10.1109/IEMECON.2017.8079587

[24] Abinaya, M., Amudha, J. (2015). Image denoising and quality measurements using wavelet thresholding. Int. J. Sci. Innov. Eng. Technol. (IJSIET), 2: 1-6.

[25] Ismael, S.H., Mustafa, F.M., Okümüs, I.T. (2016). A new approach of image denoising based on discrete wavelet transform. In 2016 World Symposium on Computer Applications \& Research (WSCAR), pp. 36-40. https://doi.org/10.1109/WSCAR.2016.30

[26] Golilarz, N.A., Demirel, H. (2017). Image de-noising using un-decimated wavelet transform (UWT) with soft thresholding technique. In 2017 9th International Conference on Computational Intelligence and Communication Networks (CICN), pp. 16-19. https://doi.org/10.1109/CICN.2017.8319347

[27] Koranga, P., Singh, G., Verma, D., Chaube, S., Kumar, A., Pant, S. (2018). Image denoising based on wavelet transform using visu thresholding technique. International Journal of Mathematical, Engineering and Management Sciences, 3(4): 444-449.

[28] Xi, J., Li, T. (2019). Image denoising method based on improved wavelet threshold transform. In 2019 IEEE Symposium Series on Computational Intelligence (SSCI), pp. 1064-1067. https://doi.org/10.1109/SSCI44817.2019.9002923

[29] Mittal, N., Raj, A., Goyal, H. (2019). Enhancement and removal of noise from medical images by wavelet transform method. In 2019 3rd International conference on Electronics, Communication and Aerospace Technology (ICECA), pp. 1126-1130. https://doi.org/10.1109/ICECA.2019.8821979

[30] Pandey, A., Singh, K.K. (2015). An overview of image denoising and image denoising techniques. Advanced Research in Electrical and Electronic Engineering, 2(9).

[31] Zhang, X. (2016). Image denoising using local Wiener filter and its method noise. Optik, 127(17): 6821-6828. https://doi.org/10.1016/j.ijleo.2016.05.002

[32] Arazm, N., Sahab, A., Kazemi, M.F. (2017). Noise reduction of SEM images using adaptive Wiener filter. In 2017 IEEE International Conference on Cybernetics and Computational Intelligence (CyberneticsCom), pp. 50-55.

https://doi.org/10.1109/CYBERNETICSCOM.2017.831 1683

[33] Devi, R., Sujatha, P. (2018). Enhancement of fingerprint image using wiener filter. International Journal of Engineering \& Technology, 7(1.1): 206-212.

[34] Metri, S., Asha, T. (2018). Patch based wiener filter for image denoising. In 2018 International Conference on Computational Techniques, Electronics and Mechanical 
Systems

(CTEMS),

pp.

392-396

https://doi.org/10.1109/CTEMS.2018.8769307

[35] Zhang, S., Li, X., Zhang, C. (2018). Modified adaptive median filtering. In 2018 International Conference on Intelligent Transportation, Big Data \& Smart City (ICITBS), pp. 262-265. https://doi.org/10.1109/ICITBS.2018.00074

[36] Tang, J., Wang, Y., Cao, W., Yang, J. (2019). Improved adaptive median filtering for structured light image denoising. In 2019 7th International Conference on Information, Communication and Networks (ICICN), pp. 146-149. https://doi.org/10.1109/ICICN.2019.8834974

[37] Erkan, U., Gökrem, L., Enginoğlu, S. (2019). Adaptive right median filter for salt-and-pepper noise removal. Uluslararası Mühendislik Araştırma ve Geliştirme Dergisi, 11(2): 542-550. https://doi.org/10.29137/umagd.495904

[38] Bouhali, A., Berkani, D. (2017). Combination of spatial filtering and adaptive wavelet thresholding for image denoising. International Journal of Image, Graphics \& Signal Processing, 9(5): 9-19. https://doi.org/10.5815/ijigsp.2017.05.02

[39] Lyakhov, P.A., Orazaev, A.R., Chervyakov, N.I., Kaplun, D.I. (2019). A new method for adaptive median filtering of images. In 2019 IEEE Conference of Russian Young Researchers in Electrical and Electronic Engineering (EIConRus), pp. 1197-1201. https://doi.org/10.1109/EIConRus.2019.8657050

[40] Sultan, N.H. (2016). Hybrid image denoising using wiener filter with discrete wavelet transform and framelet transform. Kufa Journal of Engineering, 7(2).

[41] Mridula, S., Mohanan, P. (2016). Speckle noise reduction in images using Wiener filtering and adaptive Wavelet thresholding. In 2016 IEEE region 10 conference (TENCON), pp. 2860-2863. https://doi.org/10.1109/TENCON.2016.7848566

[42] Diwakar, M., Kumar, M. (2016). Edge preservation based CT image denoising using Wiener filtering and thresholding in wavelet domain. In 2016 Fourth International Conference on Parallel, Distributed and Grid Computing (PDGC), pp. 332-336. https://doi.org/10.1109/PDGC.2016.7913171

[43] Chithra, K., Santhanam, T. (2017). Hybrid denoising technique for suppressing Gaussian noise in medical images. In 2017 IEEE International Conference on Power, Control, Signals and Instrumentation Engineering (ICPCSI), pp. 1460-1463. https://doi.org/10.1109/ICPCSI.2017.8391954

[44] Ramadhan, A., Mahmood, F., Elci, A. (2017). Image denoising by median filter in wavelet domain. arXiv preprint arXiv:1703.06499.

[45] Longkumer, M., Gupta, H. (2018). Denoising of images using wavelet transform, Weiner filter and soft thresholding. International Research Journal of
Engineering and Technology (IRJET), 5(6).

[46] Aghabalaei, A., Amerian, Y., Ebadi, H., Maghsoudi, Y. (2018). Speckle noise reduction of time series SAR images based on wavelet transform and Kalman filter. In IGARSS 2018-2018 IEEE International Geoscience and Remote Sensing Symposium, pp. 625-628. https://doi.org/10.1109/IGARSS.2018.8519431

[47] Choi, H., Jeong, J. (2018). Despeckling images using a preprocessing filter and discrete wavelet transform-based noise reduction techniques. IEEE Sensors Journal, 18(8): 3131-3139. https://doi.org/10.1109/JSEN.2018.2794550

[48] Dass, R. (2018). Speckle noise reduction of ultrasound images using BFO cascaded with wiener filter and discrete wavelet transform in homomorphic region. Procedia Computer Science, 132: 1543-1551. https://doi.org/10.1016/j.procs.2018.05.118

[49] Tayade, P.M., Bhosale, S.P. (2018). Medical Image Denoising and Enhancement using DTCWT and Wiener filter. International Journal of Advance Research, Ideas and Innovations in Technology (IJARIIT), 4(4).

[50] Luo, P., Qu, X., Qing, X., Gu, J. (2018). CT image denoising using double density dual tree complex wavelet with modified thresholding. In 2018 2nd International Conference on Data Science and Business Analytics (ICDSBA), pp. 287-290. https://doi.org/10.1109/ICDSBA.2018.00-38

[51] Qian, Y. (2018). Image denoising algorithm based on improved wavelet threshold function and median filter. In 2018 IEEE 18th International Conference on Communication Technology (ICCT), pp. 1197-1202. https://doi.org/10.1109/ICCT.2018.8599921

[52] Qian, Y. (2019). Removing of Salt-and-pepper Noise in Images Based on Adaptive Median Filtering and Improved Threshold Function. In 2019 Chinese Control and Decision Conference (CCDC), pp. 1431-1436. https://doi.org/10.1109/CCDC.2019.8832612

[53] Nishu, I.Z., Tanjim, M.F., Badrudduza, A.S.M., Al Mamun, M. (2019). A New Image Despeckling Method by SRAD Filter and Wavelet Transform Using Bayesian Threshold. In 2019 International Conference on Electrical, Computer and Communication Engineering (ECCE), pp. 1-6. https://doi.org/10.1109/ECACE.2019.8679494

[54] Fan, W.Q., Xiao, W.S. (2019). Image denoising based on wavelet thresholding and Wiener filtering in the wavelet domain. The Journal of Engineering, 2019(19): 60126015.

[55] Ferzo, B.M., Mustafa, F.M. (2020). Image Denoising in Wavelet Domain Based on Thresholding with Applying Wiener Filter. In 2020 International Conference on Computer Science and Software Engineering (CSASE), pp.

106-111. https://doi.org/10.1109/CSASE48920.2020.9142091 